\title{
Prof. Dr. W. Jadassohn zum 70. Geburtstag
}

\section{$3 / 4 \mathrm{Wt}$}

Prof. Dr. W. Jadassohn zum 70. Geburtstag

Lieber Freund und Jubilar,

Deine stete rege Tätigkeit kennt auch an der Schwelle eines neuen Dezenniums keine Rast. Aber alle, die Dir nahestehen, möchten in diesem Moment etwas verweilen, um Dir neben ihren Glückwünschen auch für Dein bisheriges und zukünftiges Schaffen herzlich zu danken. Du betrachtest zwar Dein Tun bescheiden als selbstverständliche Pflichterfüllung, darum sei dem Freunde erlaubt, rückschauend auf persönliches Erleben, heute noch mehr darüber auszusagen. Gut vier Jahrzehnte berühren sich schon unsere Wege, und eine Fülle lebendiger Erinnerungen bewahre ich von dieser Zeitspanne. Bilder tauchen vor mir auf von den gemeinsamen Zürcher Jahren mit den täglichen Laboratoriumsgesprächen oder den Arbeits-Feieraben-den in Deinem Heim bis zu den spätern Besuchen in Genf, wo jeder Abschied Deine Frage nach dem Wiederkommen einschloss. Ich möchte jetzt aber solche Wegetappen nicht einfach registrieren, ob-wohl ich damit einem Deiner obersten Grundsätze folgen würde, nein, ich darf dabei getrost auch zur Synthese gelangen, denn alle Einzel-bilder fügen sich vollkommen zusammen und runden sich zum Bild von Dir, wie wir Dich als vorbildlichen Forscher und treuen Freund kennen.

Deine seit je gehegte stifle Liebe zur Chemie hat Dich zum «Kolle-gen von der anderen Fakultät» geführt und die Basis unserer Ge-meinschaft geschafien. Ich wage sie kaum Symbiose zu nennen, denn ich fühle mich dabei viel mehr als Empfangender denn als Gebender. Deine Dich stetig umsorgende Gattin schuf einen Hort zur unge-störten Versenkung in alle uns bewegenden Probleme.

Ich sehe jene Abende vor mir, an denen wir Versuche planten oder deren Ergebnisse besprachen und formulierten. Neben uns verband derweil Deine Gattin mit emsiger Nadel schöne Farben zum Gobelin. War das nicht wie ein Beispiel, das zeigte, was Ausdauer, sinnvolles Legen der Fäden und richtiges Setzen der Lichter vermag ? - Hier durfte ich auch noch Deinen verehrten Vater erleben, dessen Wesen durch Dich in mir wach blieb. Du hast es gehegt und das Deinige vollendet geformt.

Bescheidenheit, stete Hilfsbereitschaft und mutiges Einstehen für Wahrheit und Recht sind Dir oberstes Gebot. Dem Schüler bereit-

324 Schaaf, Prof. Dr. W. Jadassohn zum 70. Geburtstag

willig und selbstlos Rat und Fiihrung bei ernsthafter Forschung zu geben, das Wissen des andern zu achten, auch in der Kritik Güte und Verstehen walten zu lassen, unbeirГt von Schwierigkeiten mit nie erlahmender Energie einen Gedanken unentwegt weiter zu verfolgen, das waren und sind Dir verpflichtende Richtlinien, die all Dein Tun leiten.

Durch das Experiment willst Du zu sicheren Ergebnissen gelangen, und die Mehrung solcher Erkenntnisse stellst Du über die Entwick-lung von Theorien. Mit einem Goethewort hast Du das selbst schon einmal treffend umschrieben. Darum haben Dich auch vor Jahren die Bienenversuche des Biologen v. Frísch so begeistert. Dort fanden sich klare Fragestellungen, auf 
die einfache Versuchsanlagen eindeutige Antworten lieferten. Das ist es, was auch Deine Forschung auszeich-net und ihren Ergebnissen die fundamentale Bedeutung verleiht.

Du tummelst Dich wahrlich auf grüner Weide und siehst eine Fülle von Problemen, die Dir keine Ruhe lassen, bis sich schöpferische Ideen geformt haben. Verschiedenes beschäftigt Deinen Geist zu gleicher Zeit. Wenn sich unvermittelt in ein Gespräch oder in den schweigenden Genuss einer Pfeife die Frage einschiebt: Kann man nicht? - dann wird offenbar, um was Deine Gedanken noch kreisen. Selbst dem vertrautern Zuhöhrer fällt dabei oft das Aufnehmen des Fadens nicht leicht, besonders wenn solche Zeichen Deiner zur Ge-staltung drängenden Phantasie durch ihre Kühnheit ihn mit der Ant-wort zaudern lassen. Ist vielleicht aber das zur Diskussion gestellte Problem Kind Deines nie versiegenden Humors, dann erscheint bei ernsthaftem Eingehen der Schalk in Deinen Augen.

Die Wesenszüge, die Dir als Forscher eigen sind, haben auch in der Freundschaft uneingeschränkt Geltung. Du kümmerst Dich mit regem Anteil um alles, was den Freund bewegt, lässest ihn an alien Deinen Erfahrungen teilhaben und kargst nicht mit gutem Rat. Wer Dich Freund nennen darf, der weiss, dass er immer mit vollem Ver-trauen zu Dir kommen und sich stets auf Deine Freundestreue ver-lassen kann.

Um Dir ein Zeichen unserer Erkenntlichkeit zu geben, ist es ein Anliegen aller, Dir einen freudigen Tag zu bereiten und für die Zu-kunft alles Gute zu wünschen.

Dein Fritz Schaaf

Lieber Herr Jubilar,

De $\Gamma$ schriftordnende Médecin adjoint erachtete es als geziemend, dass nach den Zeilen des gleichaltrigen FГeundes ein Wort aus den Reihen Ihrer auch schon ergrauten Schüler zu Ihrem Jubiläum an Sie gerichtet werde. Zu diesem Behufe darf ein Praktiker sich sozusagen aus der Froschperspektive melden.

Dass dem so sein darf, beruht auf einer Sie kennzeichnenden 1·ie-benswerten Eigenschaft: Ihr väterlich führender Rat hat Ihren Adepten, die sich der dermatologischen Exekutive bemühen, immer zur Verfügung gestanden, und für Frage und Diskussion standen und stehen Sie immer in Bereitschaft. Über ein immenses Wissen zu ver-fügen und aus diesem Schatze abgeben zu können sind zwei ganz verschiedene Dinge, die nicht in ein und derselben Persönlichkeit realisiert sein müssen. Bei Ihnen finden wir diese Qualitäten in glücklicher Art gepaart. Sie werden es an einem Ehrentage, wie wir ihn heute feiern, am ehesten zu tragen wissen, dass der Dank einmal aufs Papier muss. Fusst doch mein tägliches Tun heute noch auf der fachlichen Ernäh-rung und Erziehung - um nicht zu sagen Domestikation -, die Sie dem DermatologenEmbryo in den dreissiger Jahren haben angedeihen lassen. Durch Sie bekam ich Denken und Wissen Ihres verehrten Vaters und unsres Chefs Bruno Block eingeflösst, so dass ich noch einen guten Rest «alter Schule» mein eigen nenne.

Viel aufzunehmen gab es auf den Stationsvisiten an der Blochschen Klinik. Die bei solchem Anlass zwischen Chef und Oberarzt gepfloge-nen Diskussionen betrafen ja bald Probleme aus der vordersten Front unserer Wissenschaft, bald auch Menschliches und Allzu-menschliches, Zwiegespräche, die für den grünen Assistenzarzt eine Fundgrube bedeuteten.

Eine weitere Phase brachte der Krieg, da Sie und ich schon in der Praxis standen. Wenn Sie zum Luftschutzdienst eingerückt waren, durfte ich Sie vertreten, lernte, wie Sie Ihre Patienten betreuten und wir gerieten nach Ihrer Rückkehr ins Zivil in epikritische Diskussionen, bei denen mir viel Wissen und manche Korrektur zuteil wurden.

326 Diem, Frof. Dr. W. Jadassohn zum 70. Geburtstag 
Wenn ich Sie einmal fragte, ob Sie mir in einer dunkeln dermatolo-gischen Frage mein Wissen erhellen könnten, sahen Sie mich nicht selten leise ungehalten an. Sie formulierten die Antwort, indem Sie - wie Sie sagten - Ihren Vater zitierten, der angesichts dieser Frage gesprochen hätte: «Das möchte ich auch gerne wissen.»

Daran habe ich Ihren Respekt vor den Lücken unserer dermatolo-gischen Naturwissenschaft erfassen gelernt. Wenn Sie ein Nichtwissen zugestanden, dann gab es eben kein Wissen. Mit Spekulationen haben Sie meine Schülergeneration nie und sicher auch nicht Ihre späteren Alumnen abgespiesen.

Es war nie Ihr spontanes Anliegen, «grosse Auslegeordnung» Ihres Wissens vorzutragen, selbst wenn man Sie schüchtern darum bat. Methodisch steht fur Sie das gewissenhafte Bemühen um die Beant-wortung kritischer Fragestellungen an erster Stelle. Daraus ent-wickelten Sie ein Leben lang die experimentelle Untersuchung, mit deren gezielter Anwendung Sie scheinbar kleine faszinierende Pro-blemstellungen in grosse Zusammenhänge zu bringen verstanden. -Aber auch jede Sprechstunde blieb für Sie eine versteckte experimentelle Situation. Wenn es je einem auch in der Praxis tätigen Wissen-schaftler gelang, «dogmatischen Haltes» zu entraten, dann darf ich es von Ihnen sagen. Dieses Vorbildes heute zu gedenken war mein Anliegen.

Nehmen Sie schlichten herzlichen Dank von Ihrem

Ernst Diem, Zurich

La Clinique Universitaire de Dermatologie de Geneve tient à re-mercier tout spécialement les auteurs qui ont bien voulu honorer leur chef en lui dédiant un travail à Гoccasion de son soixante-dixième anniversaire. Ils remercient également le Professeur R. Schuppli, rédacteur de Dermatologica, ainsi que $\Gamma$ Editeur, le Docteur F. Karger, qui leur ont facilité au maximum leur tâche.

R. Paíllard, N. Hunziker, R. Brun, A.

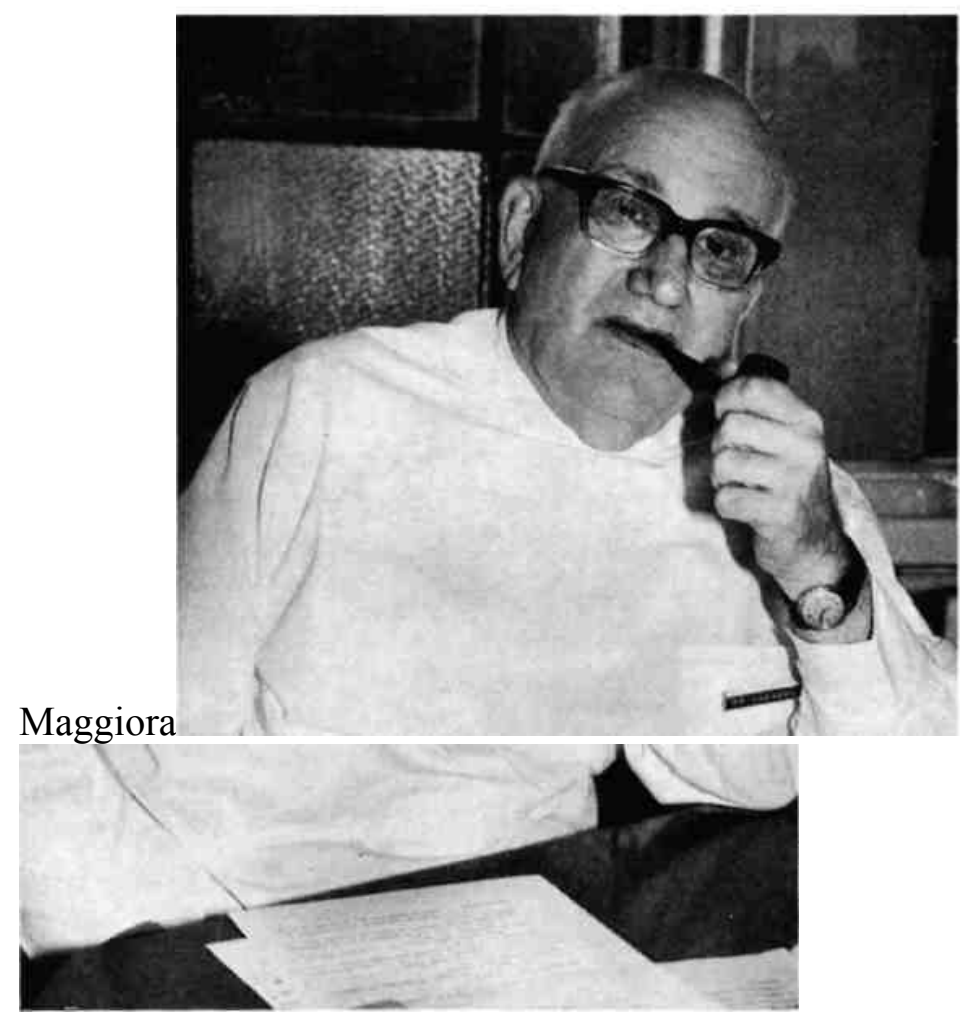

